Supporting information

\title{
Shape-Adaptive, Self-Healable Triboelectric Nanogenerator with Enhanced Performances by Soft Solid-Solid Contact Electrification
}

Yanghui Chen ${ }^{1,2,4}$, Xiong Pu ${ }^{1,2,3,{ }^{*}, \text { Mengmeng Liu }}{ }^{1,2}$, Shuangyang Kuang ${ }^{1,2}$, Panpan Zhang ${ }^{1,2}$,

Qilin Hua ${ }^{1,2}$, Zifeng Cong ${ }^{1,2}$, Wenbin Guo ${ }^{1,2}$, Weiguo Hu ${ }^{1,2,3, *}$, Zhong Lin Wang ${ }^{1,2,3,5, *}$

1. CAS Center for Excellence in Nanoscience, Beijing Key Laboratory of Micro-Nano

Energy and Sensor, Beijing Institute of Nanoenergy and Nanosystems, Chinese Academy of Sciences, Beijing 100083, China

2. School of Nanoscience and Technology, University of Chinese Academy of Sciences,

Beijing 100049, China

3. Center on Nanoenergy Researh, School of Physical Science and Technology, Guangxi University, Nanning 530004, China

4. Institute of Semiconductors, Chinese Academy of Sciences, Beijing 100083, China

5. School of Materials Science and Engineering, Georgia Institute of Technology, Atlanta, Georgia 30332, USA

*E-mail: puxiong@binn.cas.cn; *E-mail: huweiguo@binn.cas.cn; *E-mail: zlwang@gatech.edu. 


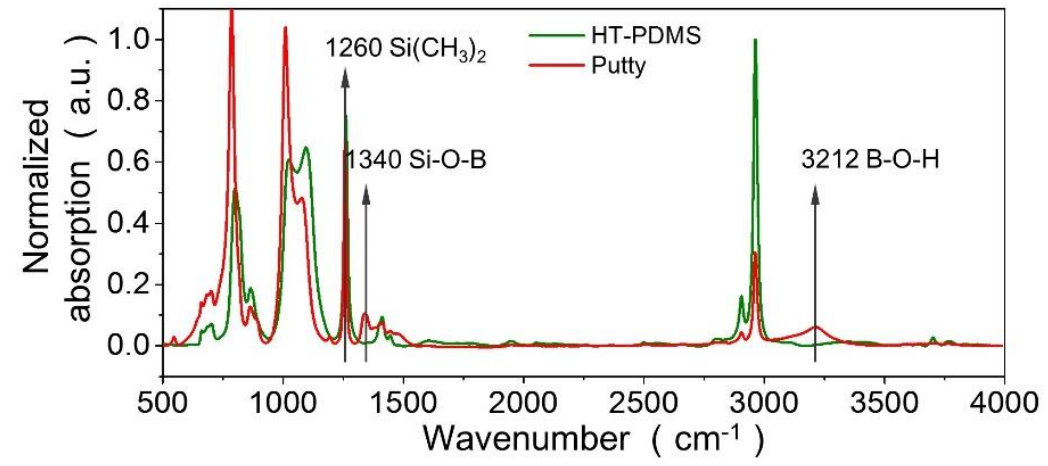

Figure S1. FTIR spectra of hydroxyl-terminated polydimethylsiloxane (HT-PDMS) and putty. The vibration at $1340 \mathrm{~cm}^{-1}$ can be assigned to the B-O-Si stretching vibration demonstrating successful cross linking of the HT-PDMS with the boric acid.

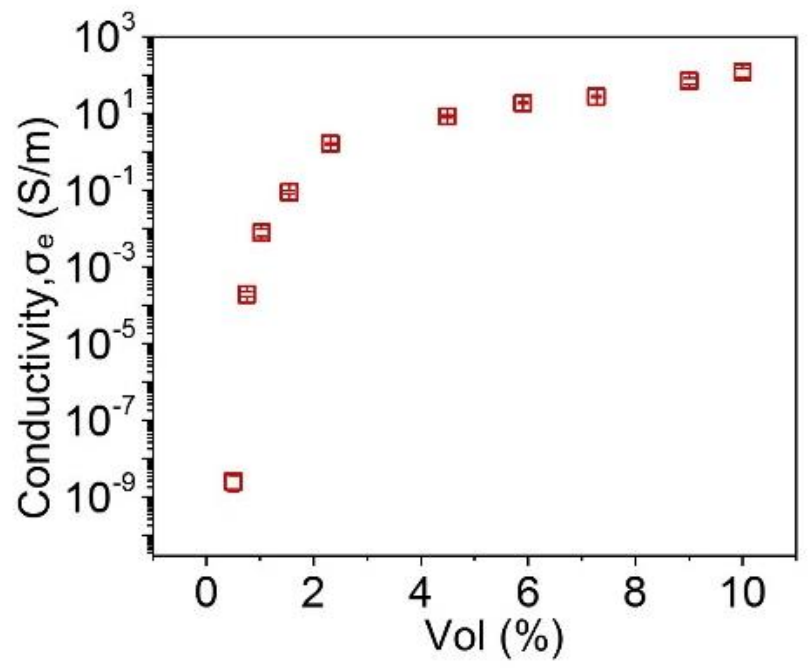

Figure S2. Electrical conductivity of CNT-Putty as a function of CNTs volume fraction.
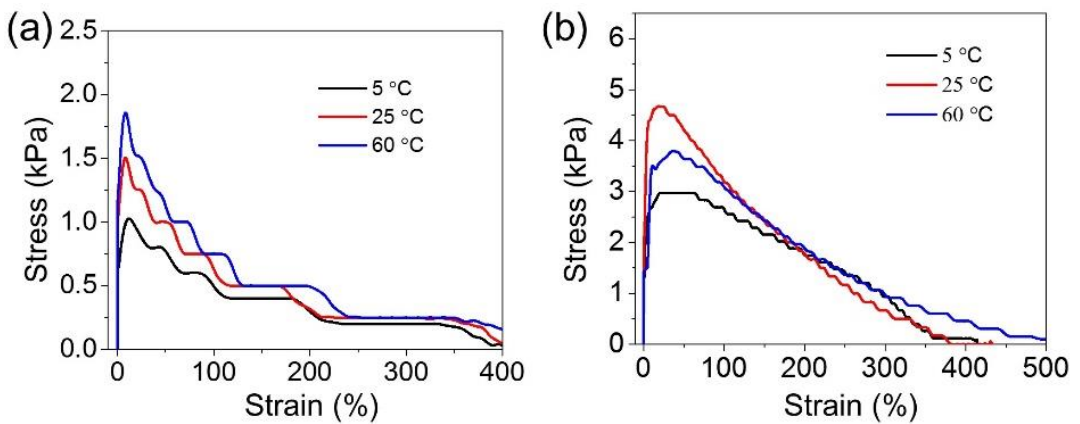
Figure S3. Tensile stress-strain curves of (a) putty and (b) CNT-putty at different temperature.
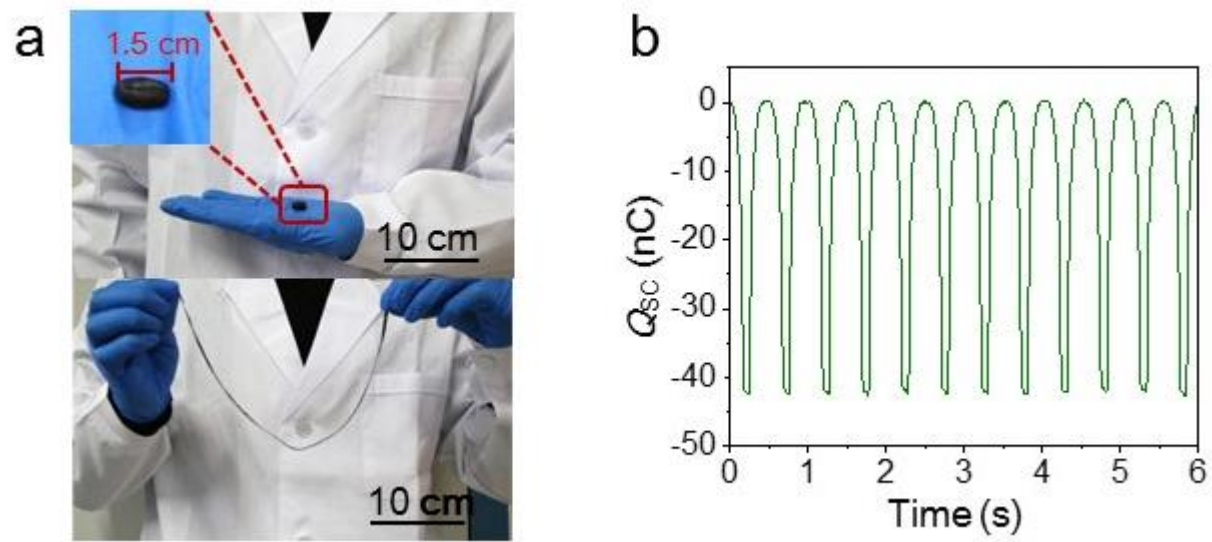

Figure S4. (a) Photographs demonstrating the stretchability of CNT-putty. (b) The transferred charges under short-circuit condition $\left(Q_{\mathrm{SC}}\right)$ upon tapping at a frequency of $\sim 2 \mathrm{~Hz}$.

Table S1. The performance comparison among shape adaptive or self-healable TENGs

\begin{tabular}{|c|c|c|c|c|c|}
\hline $\begin{array}{l}\text { Key Materials } \\
\text { of the TENGs }\end{array}$ & $\begin{array}{l}\text { Open-circuit } \\
\text { voltage }(\mathrm{V}) \\
\text { and area }\left(\mathrm{cm}^{2}\right)\end{array}$ & $\begin{array}{l}\text { Power } \\
\text { density } \\
\left(\mathrm{mW} / \mathrm{m}^{2}\right)\end{array}$ & $\begin{array}{l}\text { Work mode } \\
\text { (single-electrode } \\
\text { or two-electrod) }\end{array}$ & $\begin{array}{l}\text { Shape adaptive or } \\
\text { self-healable }\end{array}$ & Ref. \\
\hline Wavy-Kapton & $700 \mathrm{~V}, 40 \mathrm{~cm}^{2}$ & 5000 & Two-electrode & Shape adaptive & 1 \\
\hline Liquid metal & $5 \mathrm{~V}, 6.4 \mathrm{~cm}^{2}$ & 200 & Two-electrode & Shape adaptive & 2 \\
\hline PDMS-PU & $20 \mathrm{~V}, 24 \mathrm{~cm}^{2}$ & 6 & Two-electrode & Self-healable & 3 \\
\hline Slime & $50 \mathrm{~V}, 4 \mathrm{~cm}^{2}$ & 400 & Single-electrode & Self-healable & 4 \\
\hline Healable PDMS & $94 \mathrm{~V}, 6 \mathrm{~cm}^{2}$ & 327 & Single-electrode & Self-healable & 5 \\
\hline Vitrimer & $26 \mathrm{~V}, 4 \mathrm{~cm}^{2}$ & 73 & Single-electrode & $\begin{array}{l}\text { Shape adaptive } \\
\text { and self-healable }\end{array}$ & 6 \\
\hline Vitrimer & $50 \mathrm{~V}, 9 \mathrm{~cm}^{2}$ & N/A & Single-electrode & Self-healable & 7 \\
\hline Putty & $140 \mathrm{~V}, 9 \mathrm{~cm}^{2}$ & 410 & Single-electrode & $\begin{array}{l}\text { Shape adaptive } \\
\text { and self-healable }\end{array}$ & $\begin{array}{l}\text { This } \\
\text { work }\end{array}$ \\
\hline
\end{tabular}



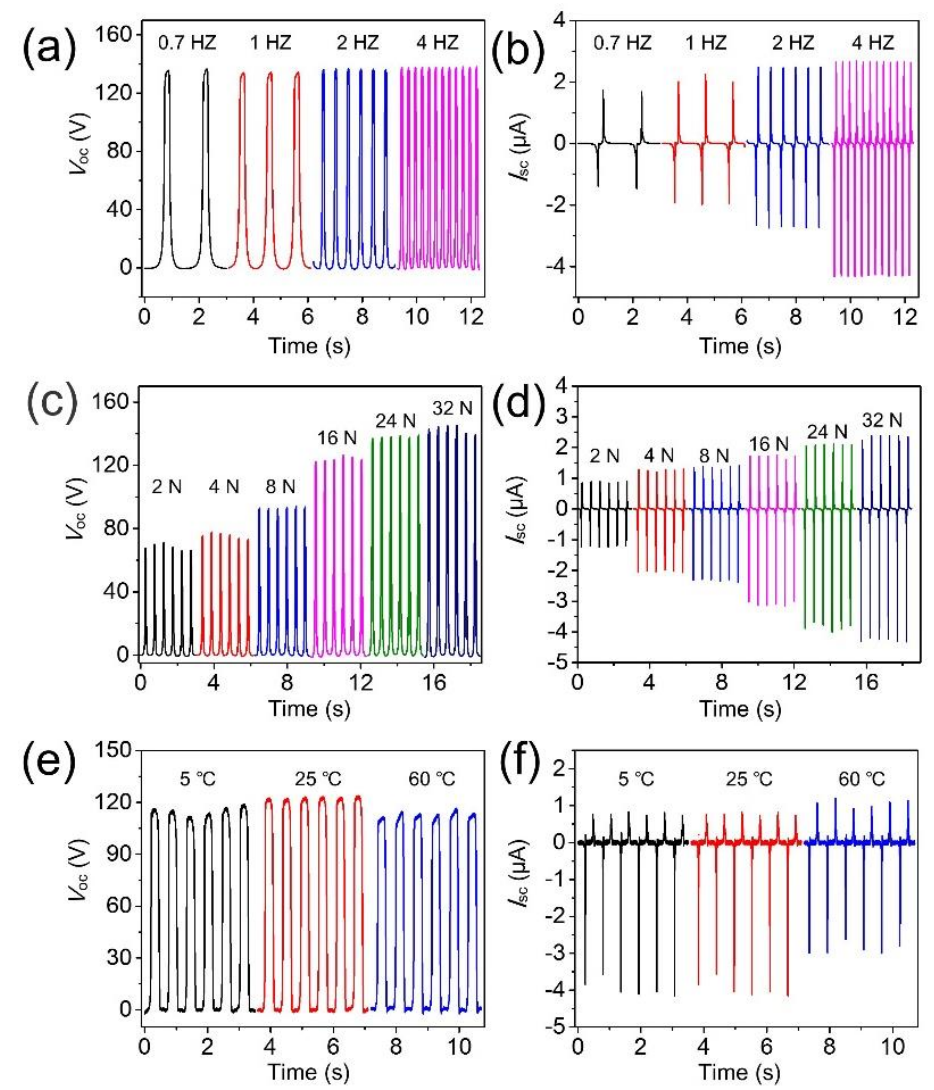

Figure S5. $V_{\mathrm{OC}}$ and $I_{\mathrm{SC}}$ curves of an SS-TENG at contact frequency $(\mathrm{a}, \mathrm{b})$, applied force (c, d), and operation temperature (e, f).
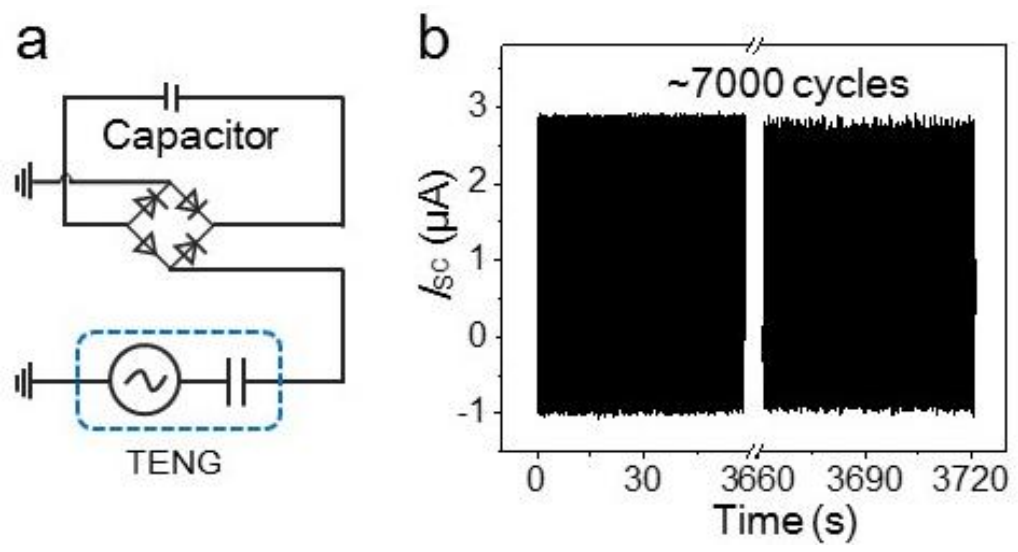

Figure S6. (a) The equivalent circuit for capacitor charging. (b) The durability of the SS-TENG for a continuous dynamic impact for $\sim 1 \mathrm{~h}$ at $2 \mathrm{~Hz}$ in single-electrode mode. 


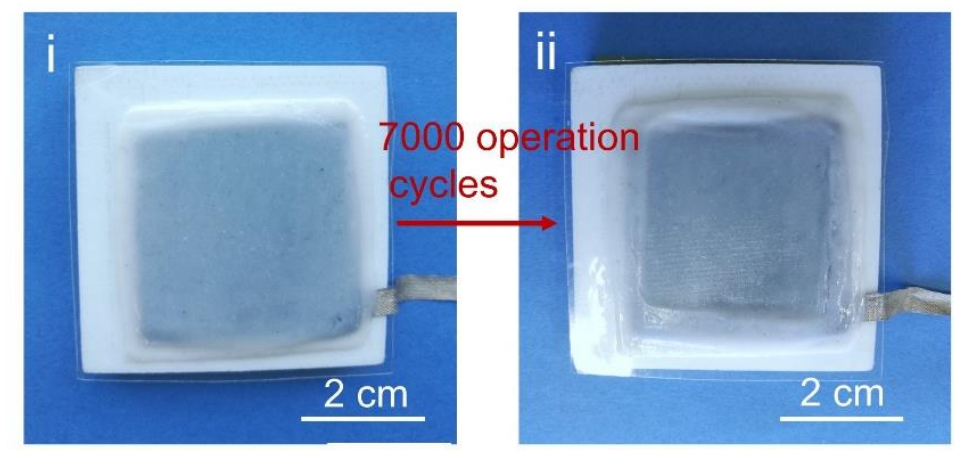

Figure S7. The photographs of the putty before (i) and after (ii) 7000 operation cycles with nickel coated fabric.
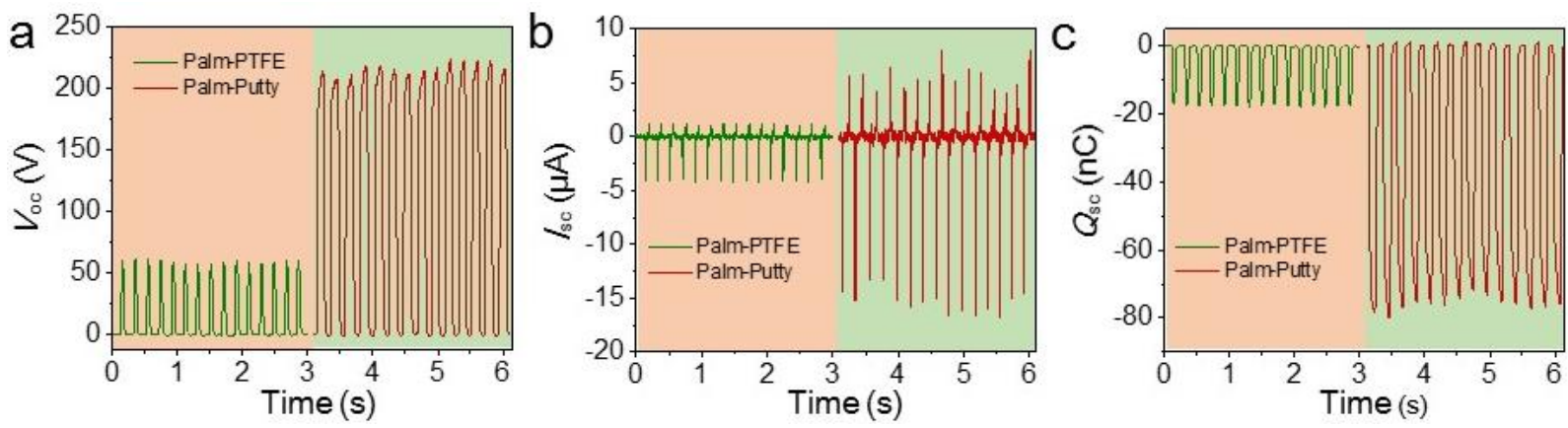

Figure S8. (a) Open-circuit voltage $\left(V_{\mathrm{OC}}\right)$, (b) short-circuit current $\left(I_{\mathrm{SC}}\right)$ and (c) transferred charges $\left(Q_{\mathrm{SC}}\right)$ of the SS-TENG mounted by palm motion.

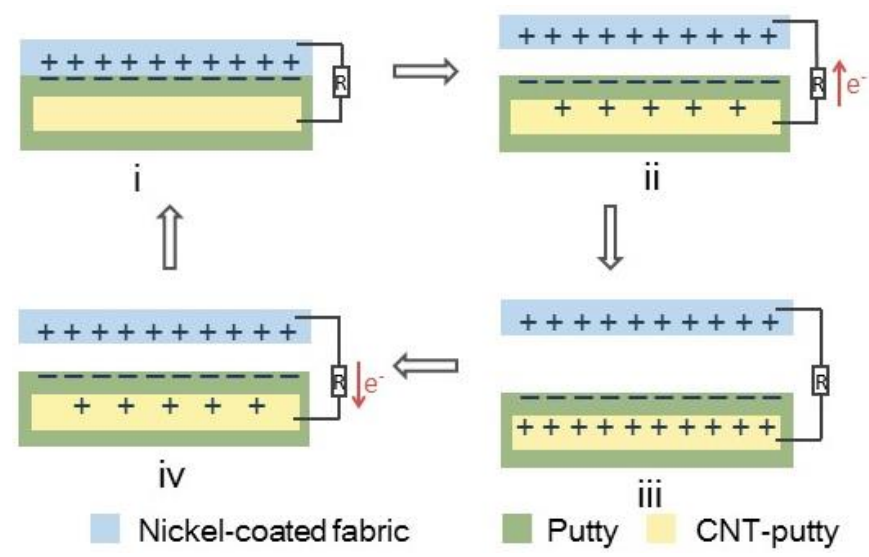

Figure S9. The scheme of the working mechanism of the TENG in two-electrode mode. 

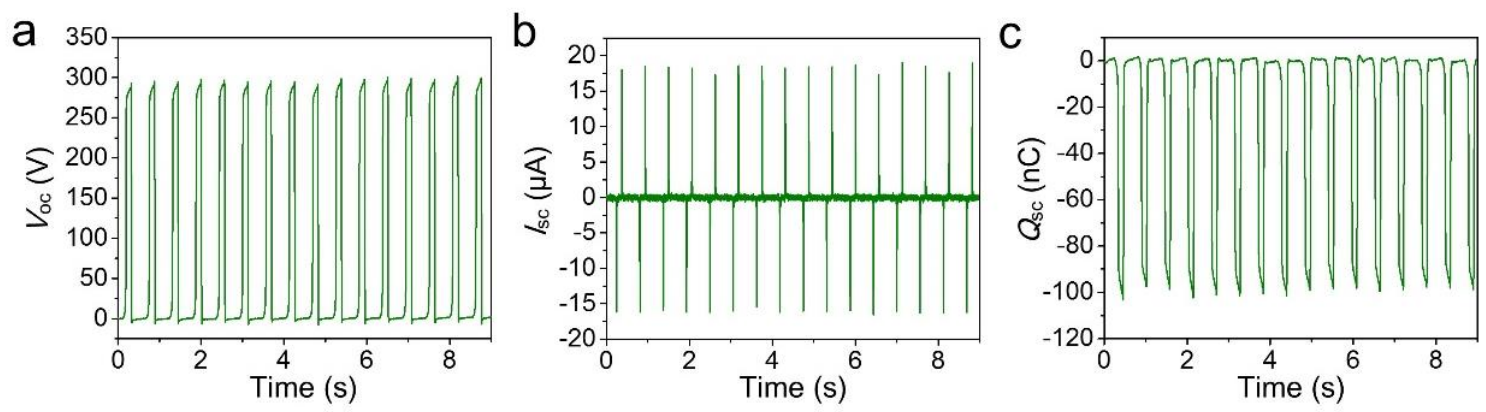

Figure 10. (a) The open-circuit voltage, (b) short-circuit current and (c) transferred charges of a TENG working at two-electrode mode.

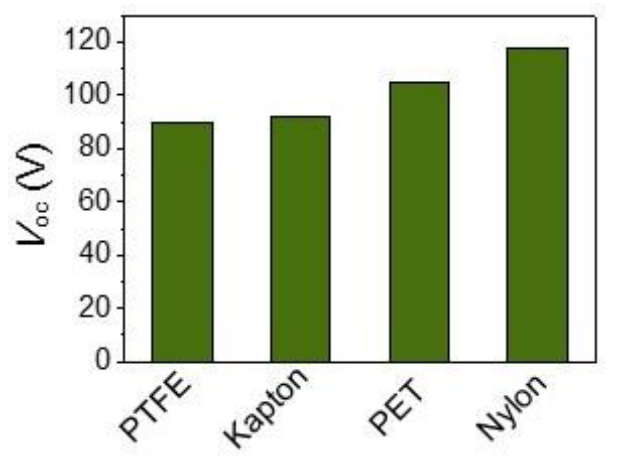

Figure S11. Summarized peak amplitude of open-circuit voltage of the SS-TENG with contact-separation motion to different materials.

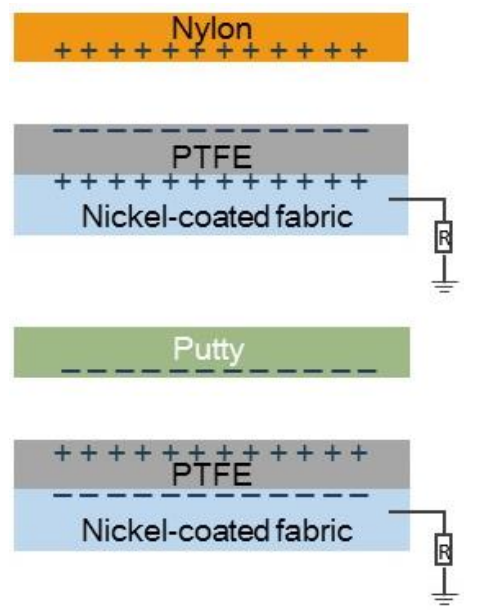

Figure S12. Structure diagram of TENGs with Nylon-PTFE and Putty-PTFE as contacting pairs, respectively. When the Nylon moving away from the PTFE film, the negative charges 
on the surface of the PTFE can induce positive charges on the electrode (Nickel-coated fabric), driving free electrons to flow from the electrode to ground. Therefore, a positive peak of the current will occur with the positive terminal of the electrometer connected to the back electrode of the PTFE contact layer. When the Nylon was reverted to approach the PTFE, a reversed output voltage/current signal will occurs.
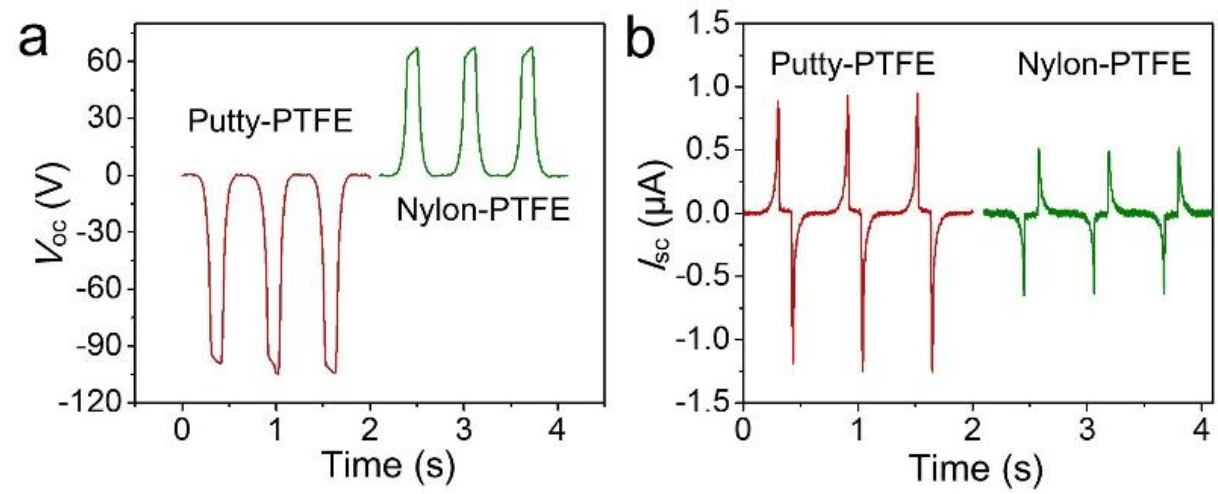

Figure S13. Output (a) $V_{\mathrm{OC}}$ and (b) $I_{\mathrm{SC}}$ of PTFE-based TENG with nylon, putty as contact layers, respectively.

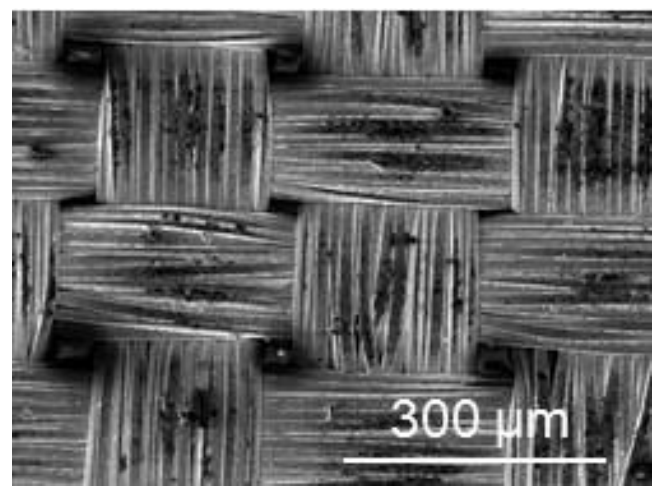

Figure S14. SEM image of the surface of the nickel coated fabric. 


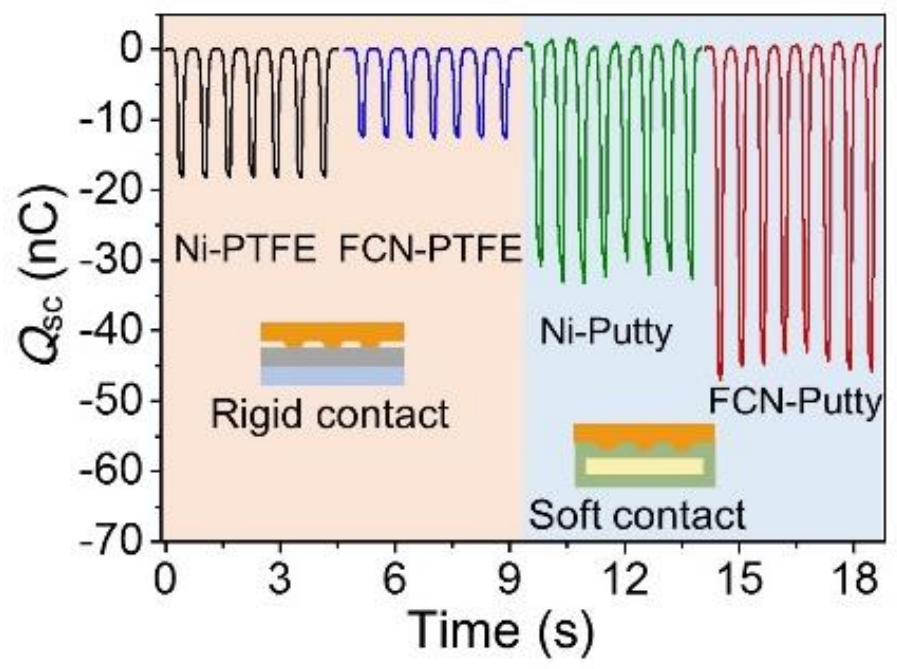

Figure S15. Transferred charges for Putty-based and PTEF-based TENG with nickel foil and nickel coated fabric (NCF) as contact layers.

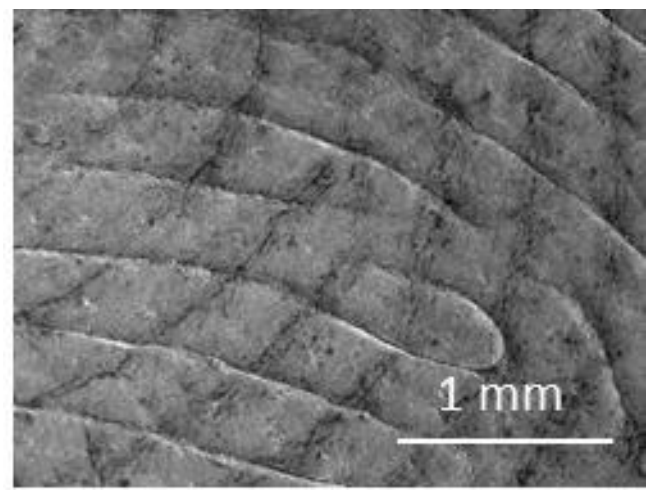

Figure S16. SEM image of the surface of the putty pressed by a finger. 


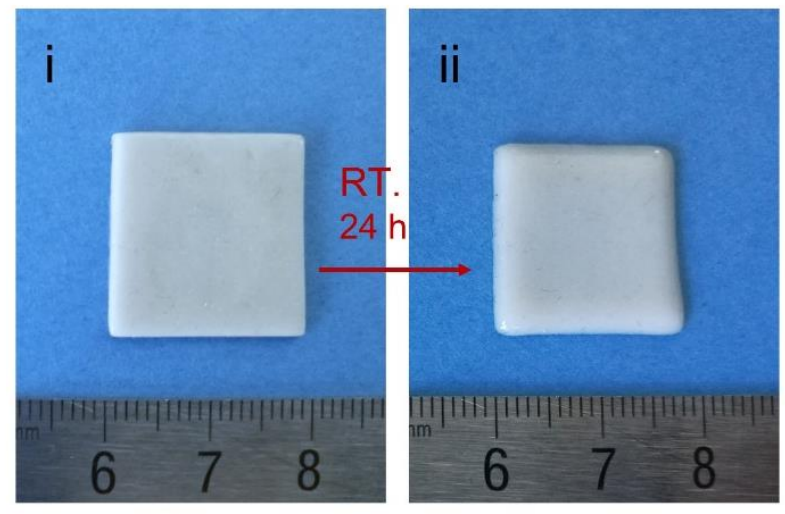

Figure S17. The photographs of the putty before (i) and after (ii) being placed at room temperature for $24 \mathrm{~h}$.

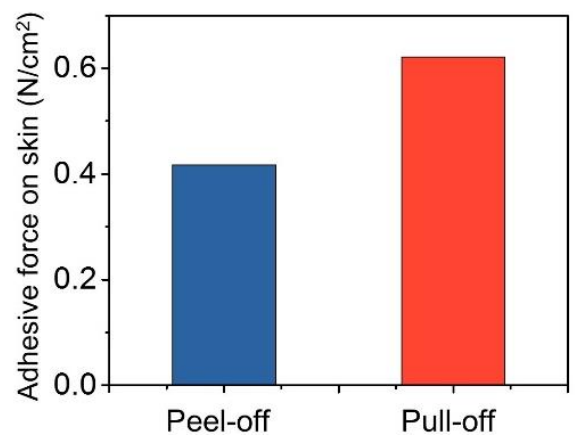

Figure S18. The adhesive force on skin measured by peeling-off and pulling-off.

(a)

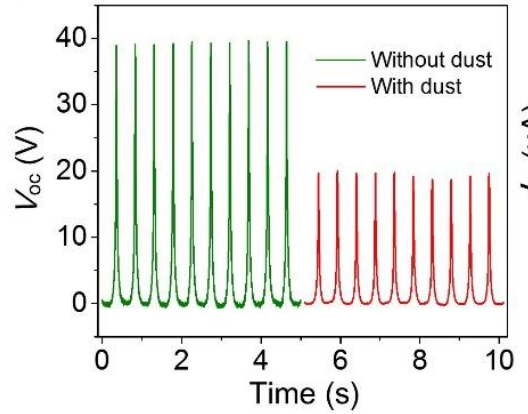

(b)

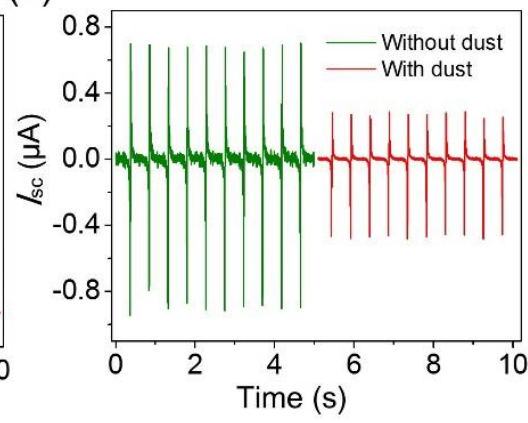

(c)

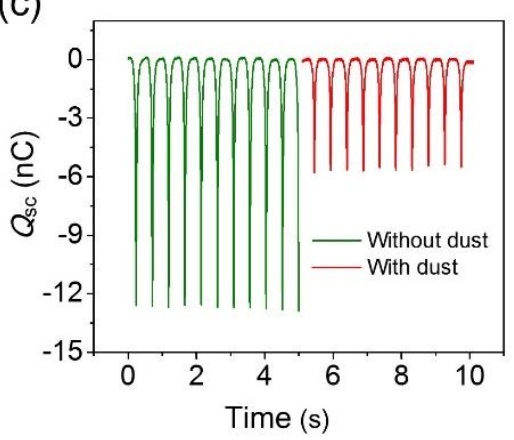

Figure S19. (a) $V_{\mathrm{OC}}$, (b) $I_{\mathrm{SC}}$ curves and (c) transferred charges of the SS-TENG with and without dust. 


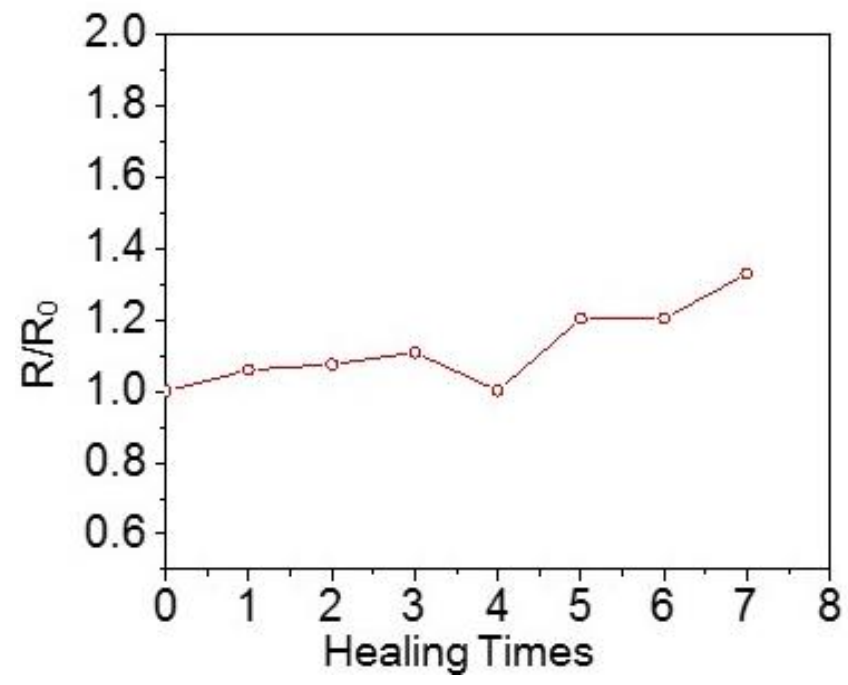

Figure S20. Resistance variation ratios of the CNT-Putty after seven cutting and healing cycles.
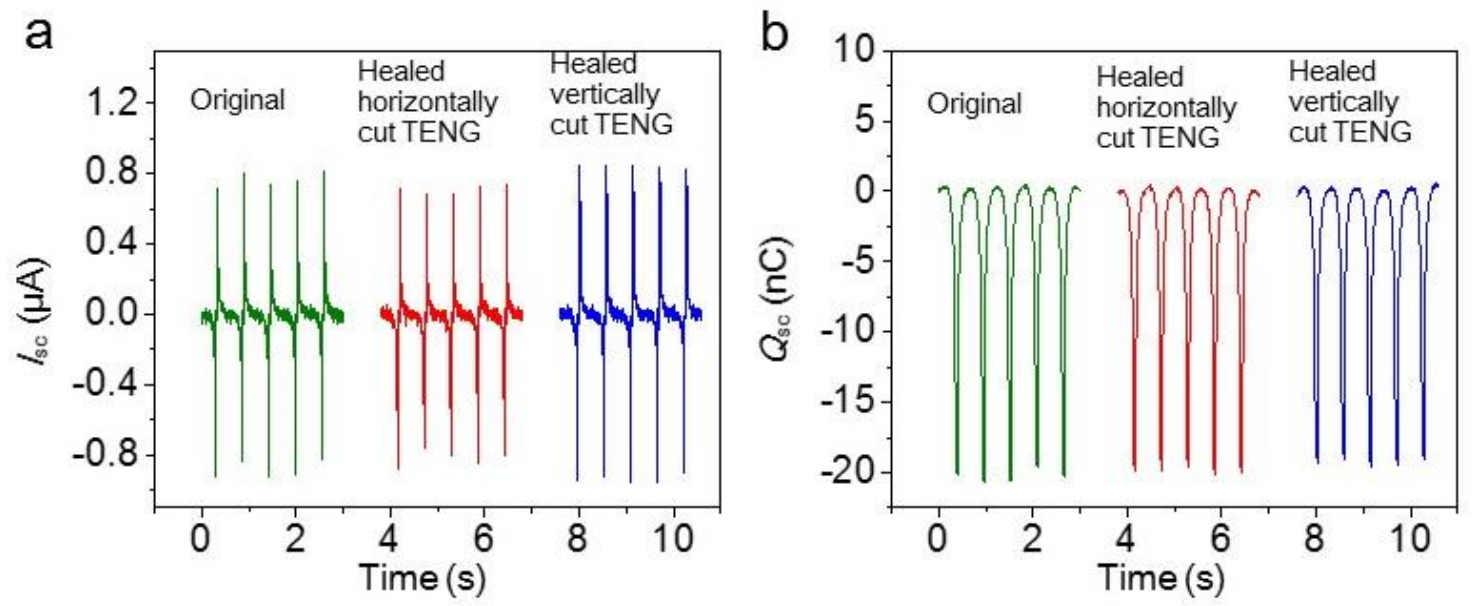

Figure S21. (a) $I_{\mathrm{SC}}$ and (b) $Q_{\mathrm{SC}}$ of the original and self-healed TENG.

References:

(1) Yang, P. K.; Lin, L.; Yi, F.; Li, X.; Pradel, K. C.; Zi, Y.; Wu, C. I.; He, J. H.; Zhang, Y.;

Wang, Z. L. A Flexible, Stretchable and Shape-Adaptive Approach for Versatile Energy

Conversion and Self-Powered Biomedical Monitoring. Adv. Mater. 2015, 27, 3817-3824. 
(2) Yi, F.; Wang, X.; Niu, S.; Li, S.; Yin, Y.; Dai, K.; Zhang, G.; Lin; L.; Wen, Z.; Guo, H.;

Wang, J.; Yeh, M.-H.; Zi, Y.; Liao, Q.; You, Z.; Zhang, Y.; Wang, Z. L. A Highly

Shape-Adaptive, Stretchable Design Based on Conductive Liquid for Energy Harvesting and Self-Powered Biomechanical Monitoring. Sci. Adv. 2016, 2, e1501624.

(3) Lee, J. H.; Hinchet, R.; Kim, S. K.; Kim, S.; Kim, S.-W. Shape Memory Polymer-Based Self-Healing Triboelectric Nanogenerator. Energy Environ. Sci. 2015, 8, 3605-3613.

(4) Parida, K.; Kumar, V.; Jiangxin, W.; Bhavanasi, V.; Bendi, R.; Lee, P. S. Highly Transparent, Stretchable, and Self-Healing Ionic-Skin Triboelectric Nanogenerators for Energy Harvesting and Touch Applications. Adv. Mater. 2017, 29, 1702181.

(5) Sun, J.; Pu, X.; Liu, M.; Yu, A.; Du, C.; Zhai, J.; Hu, W.; Wang, Z. L. Self-Healable, Stretchable, Transparent Triboelectric Nanogenerators as Soft Power Sources. ACS Nano 2018, 12, 6147-6155.

(6) Deng, J.; Kuang, X.; Liu, R.; Ding, W.; Wang, A. C.; Lai, Y.-C.; Dong, K.; Wen, Z.; Wang, Y.; Wang, L.; Qi, H. J.; Zhang, T.; Wang, Z. L. Vitrimer Elastomer-Based Jigsaw Puzzle-Like Healable Triboelectric Nanogenerator for Self-Powered Wearable Electronics. Adv. Mater. 2018, 30, e1705918.

(7) Guan, Q.; Dai, Y.; Yang, Y.; Bi, X.; Wen, Z.; Pan, Y. Near-Infrared Irradiation Induced Remote and Efficient Self-Healable Triboelectric Nanogenerator for Potential Implantable Electronics. Nano Energy 2018, 51, 333-339. 